UPR-599T

\title{
Measure Factors, Tension, and Correlations of Fluid Membranes
}

\author{
W. Cai, T.C. Lubensky, P. Nelson and T. Powers \\ Physics Department, University of Pennsylvania \\ Philadelphia, PA 19104 USA
}

\begin{abstract}
We study two geometrical factors needed for the correct construction of statistical ensembles of surfaces. Such ensembles appear in the study of fluid bilayer membranes, though our results are more generally applicable. The naive functional measure over height fluctuations must be corrected by these factors in order to give correct, self-consistent formulas for the free energy and correlation functions of the height. While one of these corrections the Faddeev-Popov determinant - has been studied extensively, our derivation proceeds from very simple geometrical ideas, which we hope removes some of its mystery. The other factor is similar to the Liouville correction in string theory. Since our formulas differ from those of previous authors, we include some explicit calculations of the effective frame tension and two-point function to show that our version indeed secures coordinate-invariance and consistency to lowest nontrivial order in a temperature expansion.
\end{abstract}

$1 / 94$ 


\section{Introduction and Summary}

Amphiphilic molecules in water tend to aggregate into thin flexible bilayers, which in turn form a wide variety of nearly two-dimensional structures with characteristic size of order microns [1]. The study of these structures may become significant for biology: they are certainly important technologically as the underlying elements of microemulsions and other complex fluids.

To understand the static and dynamic properties of bilayer membranes, and the transitions between different morphologies, we must first understand the role of thermal fluctuations in two-dimensional surfaces, a subtle problem in statistical mechanics. Thermal fluctuations are important because regardless how stiff a membrane may be, very longwavelength undulations in its shape are always allowed; on long enough length scales any membrane will appear flexible and will undulate significantly, a phenomenon first described in the cell walls of red blood cells in the 19th century [2]. ${ }^{1}$

Since the length scales we wish to study are much larger than the size of the constituent molecules, we expect that a membrane will be characterized by just a few effective parameters, summarizing the effects of the complicated molecular forces. Indeed, the famous Canham-Helfrich model[4] describes the equilibrium statistical mechanics of membranes in terms of just two parameters: a chemical potential for the addition of surfactant molecules, which we will call $\mu_{0}$, and a bending energy coefficient, the stiffness $\kappa_{0}$. (Another parameter, the gaussian stiffness $\bar{\kappa}_{0}$, only enters when we consider topology change.) The bare coefficients $\mu_{0}, \kappa_{0}$ are not directly observable, but from them we can derive some more relevant phenomenological parameters. We will mainly study the "frame tension" $\tau$, which is the free energy per unit area of the fluctuating membrane, and the two-point correlation $\langle h(u) h(0)\rangle$ of the height $h(u)$ of the membrane from its average plane, which we fix by fixing the edges of the membrane to lie on a square frame. The leading behavior of this two-point function at small wavenumber defines another phenomenological parameter, the " $q^{2}$ coefficient" $r$ via

$$
\langle h(q) h(-q)\rangle=\frac{A_{B} T}{r q^{2}+\mathcal{O}\left(q^{4}\right)},
$$

where $A_{B}$ is the area of the frame, $T$ is the temperature (we set Boltzmann's constant $k_{B}$ equal to one), and $q$ is a wavenumber. Another reason to introduce $r$ is that in dynamics

1 Strictly speaking we are here talking about "fluid membranes," those with no internal structure or order within the surface. In this paper we discuss only fluid membranes, but our measure factors can be used in more complicated situations too [3]. 
problems we can still calculate correlation functions like (1.1), while there is no analog of the free energy from which to compute $\tau$. Two of us study the dynamical problem in [5].

In this paper we will explore the relation between the various coefficients $\mu_{0}, \kappa_{0}, \tau$, and $r$. More generally, on long length scales the precise size of the constituent molecules is immaterial: we can get the same answers using molecules of size $a$ with coefficients $\mu_{0}$, $\kappa_{0}$ or with rescaled molecules of size $b^{-1} a, b<1$, and effective coefficients $\mu_{\text {eff }}(b), \kappa_{\text {eff }}(b)$. The scale dependence of $\kappa_{\text {eff }}$ is well known [6]-[11], but various answers for $\mu_{\text {eff }}$ have been given (for example, see [8][9][11]-[15]). In part the differences reflect convention, but there is a key physics point which we will address: to get correct answers we must define our functional measure properly. Two of us have already discussed such matters in [3], but here we will add a number of points, as follows.

We first introduce the effective action $\Gamma[\bar{h}]$ of the fluctuating membrane and recall the argument of [3] that its form is constrained by the coordinate invariance of the underlying theory. In particular we show in sect. 2 that the coefficients $r$ and $\tau$ defined above must be equal. We then set up a naive, uncorrected, calculational scheme which yields (consistent with [15])

$$
\tau=\mu_{0}+\frac{T}{2} \int^{\Lambda} \frac{\mathrm{d}^{2} q}{(2 \pi)^{2}} \log \left[\left(\mu_{0} q^{2}+\kappa_{0} q^{4}\right) \frac{a^{2} \lambda^{2}}{2 \pi T}\right],
$$

where $\Lambda=2 \pi / a$ and $a$ is the linear size of the constituent molecules. Following Morse and Milner we have introduced the thermal de Broglie wavelength

$$
\lambda=h / \sqrt{2 \pi m T}
$$

where $m$ is the mass of a surfactant molecule [16]. The same calculational scheme however yields a very different formula for $r$. In fact (1.2) is our final result, but clearly we need to work a bit harder to understand $r$.

The first correction factor, the "Faddeev-Popov" factor, is well known. In [3] we gave a formula for this factor which differs from the one given by David [14]; here in sect. 3 we give a simple geometrical motivation followed by a derivation of our formula which is simpler than the one in [3]. While unimportant in the calculation of $\tau$, this factor does modify the naive calculation of $r$ (and higher correlation functions too), yielding an $r_{\mathrm{FP}}$ which still disagrees with $\tau$.

Next in sect. 4 we argue that another correction factor is needed. Physically our membrane consists of constituent molecules each occupying a fixed area $a^{2}$ in physical 3 -space. As the surface bows outward away from its flat equilibrium configuration its area 
increases and additional molecules must be brought in from solution, effectively increasing the number of degrees of freedom in the membrane problem. A statistical measure with variable number of degrees of freedom, depending on the configuration itself, is rather complicated. We would prefer a measure with fixed number of degrees of freedom, as indeed we used implicitly in the naive derivation of $r_{\mathrm{FP}}$. Since changing the number of degrees of freedom, or equivalently the ultraviolet cutoff, can be compensated by renormalizing the bare energy, we expect that a counterterm correction will need to be added to the naive derivation.

We fix this counterterm by requiring consistency between two different Monge-gauge calculations of the free energy (in the Appendix we give a different strategy). The required counterterm turns out to make a contribution precisely changing the $q^{2}$ coefficient from $r_{\mathrm{FP}}$ to $r=\tau$.

The introduction of our second correction factor may seem like ad hoc wish-fulfillment, but in the Appendix we give some detailed calculations showing how it and the FaddeevPopov factor together are crucial to obtain consistent answers; in particular we show that the specific coefficient of the counterterm implied by consistency is the right one to get $r=\tau$. Furthermore, an analogous correction is well known in the string theory literature, where it is called the "Liouville factor" [17][18]. This factor sometimes appears in the previous membrane literature (e.g. [19]), but one gets the impression that it matters only in the low-stiffness regime beyond the persistence length; again, we will see that it is needed to get the correct correlation functions even in the more physical stiff regime. Our correction differs in detail from the Liouville factor because the latter is appropriate for

conformal gauge, but in each case the motivation is the same. We will also argue that the usual interpretation of the Liouville factor as a Jacobian is misleading; really as argued above it reflects renormalization.

Again our counterterm is important because without it, ordinary diagrammatic perturbation theory gives incorrect results due to the subtlety in the statistical measure discussed above. Finally we conclude in sect. 5 .

\section{General Arguments}

We will consider fluid membranes, those with no internal order or structure. Real membranes are typically fluid at temperatures high enough to destroy in-plane order, but in any case such order can easily be incorporated into the argument below [3]. The 
constituents of a membrane have a preferred spacing, with a high energy cost for local deviations from that density. Thus, in this regime the free energy cost of a membrane configuration depends only on its shape.

As mentioned in the Introduction, we can thus capture the physics of length scales much longer than the constituent molecule size by regarding the surface as continuous and using the Canham-Helfrich free energy [4]

$$
\mathcal{H}=\mu_{0} \int \mathrm{d} S+\frac{\kappa_{0}}{2} \int \mathrm{d} S\left(\frac{1}{R_{1}}+\frac{1}{R_{2}}\right)^{2}
$$

Here $\mathrm{d} S$ is the element of surface area, $\int \mathrm{d} S$ is the total area of the surface, and $R_{1}, R_{2}$ are the principal radii of curvature of the surface. The two coefficients $\mu_{0}, \kappa_{0}$ are respectively the area cost and bending rigidity.

The form of (2.1) is severely constrained by the requirement that the free energy depend only on the membrane's shape, and not on how one chooses to label points on the surface. While this property is manifest in (2.1), to do calculations a more explicit version of this equation proves necessary, in which we do choose coordinates $u=\left(u^{1}, u^{2}\right)$ to label points $\mathbf{R}(u)$ of the surface. As usual, we then get tangent vectors $\mathbf{e}_{a}=\partial_{a} \mathbf{R} \equiv$ $\partial \mathbf{R} / \partial u^{a}$ at $u$ and a metric tensor $g_{a b}=\mathbf{e}_{a} \cdot \mathbf{e}_{b}$ at each point. The unit normal is then $\mathbf{n}=\left(\mathbf{e}_{1} \times \mathbf{e}_{2}\right) /\left|\mathbf{e}_{1} \times \mathbf{e}_{2}\right|$, and the curvature tensor is $K_{a b}=\mathbf{n} \cdot \nabla_{a} \partial_{b} \mathbf{R}$, where $\nabla_{a}$ is the covariant derivative associated to the metric $g_{a b}$. Letting $g \equiv \operatorname{det}\left[g_{a b}\right],\left[g^{a b}\right]=\left[g_{a b}\right]^{-1}$, and $K_{a}^{a} \equiv g^{a b} K_{a b}$, eqn. (2.1) becomes

$$
\mathcal{H}=\mu_{0} \int d^{2} u \sqrt{g}+\frac{\kappa_{0}}{2} \int d^{2} u \sqrt{g}\left(K_{a}^{a}\right)^{2}
$$

If we change the coordinates from $u^{a}$ to $\widetilde{u}^{a}=\widetilde{u}^{a}(u)$ we just relabel points in the plane. For an infinitesimal change $\widetilde{u}^{a}=u^{a}+\varepsilon^{a}(u)$ we get a new $\widetilde{\mathbf{R}}(\widetilde{u})=\mathbf{R}(u)$ describing the same surface:

$$
\widetilde{\mathbf{R}}(u)=\mathbf{R}(u)-\varepsilon^{a}(u) \mathbf{e}_{a}(u)
$$

The change of $\mathbf{R}$ is a tangential motion; conversely, tangential changes of $\mathbf{R}$ are not physical changes to the shape of the surface and ought not to be counted separately in the statistical sum. We thus need to supplement (2.2) by choosing a protocol for assigning a single coordinate system to each shape, and a procedure for discarding every surface $\mathbf{R}(u)$ not parameterized in this way. Any such choice is called a "gauge-fixing" procedure. 
A popular class of gauge-fixing procedures are the so-called "normal gauges." Suppose we have arranged that configurations very close to one reference surface will dominate the statistical sum (by stretching the membrane across a fixed frame, for instance). We choose once and for all a parameterization $\mathbf{R}_{0}(u)$ for this reference surface and compute its unit normal $\mathbf{n}_{0}(u)$. Then other nearby surfaces can be written as

$$
\mathbf{R}(u)=\mathbf{R}_{0}(u)+h(u) \mathbf{n}_{0}(u)
$$

where the height field $h$ at any point is uniquely defined as the normal distance from that point to the reference surface. We thus describe each distinct surface once when we sum over all functions $h(u)$. Of course the point of this paper is that the correct definition of this sum is a tricky business, but let us proceed formally to obtain some general properties constraining the correct prescription.

Let us consider a membrane confined to span a square, flat frame of area $A_{B}$. We suppose surfactant molecules can join or leave the membrane with a fixed cost in free energy of $\mu_{0}$ per added area (one can easily pass to an ensemble with a fixed number of molecules by a Legendre transformation). The full free energy of our system $Z\left(A_{B}\right)$ then depends on the base area, and implicitly on $\mu_{0}$, the stiffness $\kappa_{0}$ appearing in $(2.1)$, the size $a \equiv 2 \pi / \Lambda$ of the constituent molecules, and the temperature $T$.

Since our frame is flat, the equilibrium (zero-temperature) surface will be flat too, and so it is convenient to work in Monge gauge, a normal gauge with $\mathbf{R}_{0}(u)$ the surface spanned by the frame, with Cartesian coordinates $u^{a}$ running from zero to $\sqrt{A_{B}}$. In addition to $Z\left(A_{B}\right)$ we will find it convenient to introduce the "effective action" $\Gamma[\bar{h}]$. To define $\Gamma[\bar{h}]$ we introduce a forcing term into $\mathcal{H}, \mathcal{H}_{j}=\mathcal{H}-\int \bar{h} j$, and adjust $j$ to ensure that $\langle h(u)\rangle$ is the desired function $\bar{h} .^{2}$ Computing the partition function

$$
Z\left[A_{B}, j\right]=\int[D h] \mathrm{e}^{-\mathcal{H} / T} \mathrm{e}^{(1 / T) \int h j}
$$

we then let

$$
\Gamma[\bar{h}] \equiv-T \log Z\left(A_{B}, j\right)+\int \bar{h} j
$$

2 This is a formal trick to obtain correlations of height; we do not imagine $j$ as coming from any physical force. Mathematically $j=j_{12} \mathrm{~d}^{2} u$ is a density on parameter space, so that $\delta / \delta j(u)$ is a scalar. For more details see [3]. 
The frame tension is the free energy per area of the unforced membrane, so we have

$$
\tau=-\frac{T}{A_{B}} \log Z\left(A_{B}\right)=\frac{1}{A_{B}} \Gamma(\bar{h}=0) \equiv \frac{1}{A_{B}} \Gamma^{(0)} .
$$

Physically there is nothing special about Monge gauge. Even if we agree to work in Monge gauge there is nothing special about a reference surface $\mathbf{R}_{0}(u)$ coinciding with the plane of the frame; a tilted reference surface must give the same free energy. All that matters is the physical area of the frame. Thus the terms of $\Gamma[\bar{h}]$ involving at most first derivatives of $\bar{h}$ (higher derivatives are insensitive to tilting) must enter in the combination $\int \mathrm{d}^{2} u \sqrt{\bar{g}}$, where

$$
\bar{g}_{a b}=\delta_{a b}+\partial_{a} \bar{h} \partial_{b} \bar{h}
$$

is the induced metric and $\bar{g}=\operatorname{det} \bar{g}_{a b}$ as usual. In other words,

$$
\Gamma[\bar{h}]=\tau \int \mathrm{d}^{2} u\left(1+\frac{1}{2}(\partial \bar{h})^{2}+\cdots\right)+\cdots,
$$

where the first ellipsis involves $\mathcal{O}\left(\bar{h}^{4}\right)$ while the second involves more derivatives than $\bar{h}$ 's. We gave a more detailed argument for this conclusion in [3].

Whatever the precise form of the measure $[D h]$ appearing in (2.5), it does not depend on $j$, and so the first moment

$$
\langle h(u)\rangle_{j} \equiv \int[D h] \mathrm{e}^{-\mathcal{H} / T} \mathrm{e}^{(1 / T) \int h j} h(u)=T Z^{-1} \frac{\delta Z[j]}{\delta j(u)} .
$$

Differentiating (2.6) and using $\langle h\rangle_{j}=\bar{h}$, we find

$$
\frac{\delta \Gamma}{\delta \bar{h}(u)}=j(u)-T Z^{-1} \int \frac{\delta j\left(u^{\prime}\right)}{\delta \bar{h}(u)} \frac{\delta Z}{\delta j\left(u^{\prime}\right)}+\int \frac{\delta j\left(u^{\prime}\right)}{\delta \bar{h}(u)} \bar{h}\left(u^{\prime}\right)=j(u)
$$

Differentiating again and using $\langle\bar{h}\rangle_{j=0}=0$, we see that

$$
\begin{aligned}
\left\langle h(u) h\left(u^{\prime}\right)\right\rangle_{j=0} & =\left.Z^{-1} T^{2} \frac{\delta^{2} Z}{\delta j(u) \delta j\left(u^{\prime}\right)}\right|_{0}=\left.Z^{-1} T \frac{\delta}{\delta j(u)}\right|_{0}\left(Z \bar{h}\left(u^{\prime}\right)\right) \\
& =\left.T \frac{\delta \bar{h}\left(u^{\prime}\right)}{\delta j(u)}\right|_{0}=T\left[\left.\frac{\delta j(u)}{\delta \bar{h}\left(u^{\prime}\right)}\right|_{0}\right]^{-1}=T\left[\left.\frac{\delta^{2} \Gamma}{\delta \bar{h}(u) \delta \bar{h}\left(u^{\prime}\right)}\right|_{0}\right]^{-1} .
\end{aligned}
$$

Thus the inverse of the two-point function is just the quadratic part of the effective action.

We have repeated the above well-known argument to make a point: It in no way depended upon the niceties of the measure $[D h]$. Any exotic gauge-fixing or cutoff-stretching 
factor which we may eventually fold into $[D h]$ will not alter the above conclusion, though they can and will affect the relation of the quadratic part to the constant part $\Gamma^{(0)}$. Combining (2.11) with (2.8), (1.1) at once shows that the $q^{2}$ coefficient $r$ equals the frame tension $\tau$.

Let us make a first attempt at calculating $r$ and $\tau$. If the temperature $T$ is small we can expand everything around the equilibrium configuration $h=0$. The energy functional (2.2) then becomes [11]

$$
\begin{aligned}
\mathcal{H} & =\mu_{0} \int \mathrm{d}^{2} u\left(1+\frac{1}{2}(\partial h)^{2}-\frac{1}{8}(\partial h)^{4}\right) \\
& +\frac{\kappa_{0}}{2} \int \mathrm{d}^{2} u\left[\left(\partial^{2} h\right)^{2}-\frac{1}{2}(\partial h)^{2}\left(\partial^{2} h\right)^{2}-2\left(\partial^{2} h\right) \partial_{a} h \partial_{b} h \partial_{a} \partial_{b} h\right]+\mathcal{O}\left(h^{6}\right)
\end{aligned}
$$

In this formula $\partial_{a}=\partial / \partial u^{a}, \partial^{2}=\delta^{a b} \partial_{a} \partial_{b}$ is the flat laplacian, and all indices are contracted with the Kronecker symbol. Thus all $h$-dependence is explicitly in view in (2.12). To calculate $Z\left(A_{B}\right)$ we need a proposal for $[D h]$. The simplest choice is to choose a mesh of grid points $\left\{u_{i j}\right\}$ on $u$ space, spaced by $a$, and let

$$
[D h]_{\text {naive }}=\prod_{i j}\left(\mathrm{~d} h\left(u_{i j}\right) / \lambda\right)
$$

We introduced the thermal wavelength $\lambda$ here to render the measure properly dimensionless. ${ }^{3}$ We will argue later that $[D h]_{\text {naive }}$ is wrong, but for now we take it as our starting point.

For low temperature we approximate $Z\left(A_{B}\right)$ as $\mathrm{e}^{-\mu_{0} A_{B} / T}$ times a gaussian integral. Collecting the quadratic terms of (2.12) and writing $\int \mathrm{d}^{2} u=a^{2} \sum_{i j}$ we find

$$
\Gamma^{(0)} \equiv-T \log Z\left(A_{B}\right)=\mu_{0} A_{B}+\frac{T}{2} \sum \log \left[\frac{\lambda^{2} a^{2}}{2 \pi T}\left(\mu_{0} q^{2}+\kappa_{0} q^{4}\right)\right] .
$$

Now the sum is over the points $q$ of reciprocal space. The upper limit of this sum is related to the constituent size $a$; for example, if in (2.13) we choose points on a square grid, then our Brillouin zone is a square of length $2 \pi / a$. Thus we recover the announced formula (1.2).

3 In [15] and [3] this scale was taken to be the molecule size $a$ or a constant multiple of it. This is legitimate when we do not care about the bare chemical potential, for example when we just want to dial $\mu_{0}$ to zero physical tension. But $\mu_{0}$ does have physical meaning, and Milner and Morse have argued that the factor in (2.13) is the correct choice [16]. 
Next we turn to the two-point function, which we provisionally define as

$$
\langle h(u) h(0)\rangle_{\text {naive }}=Z^{-1} \int[D h]_{\text {naive }} \mathrm{e}^{-\mathcal{H} / T} h(x) h(0)
$$

This time however the constant term of (2.12) drops out, the quadratic term gives the leading contribution $\langle h(q) h(-q)\rangle_{0}=\frac{T A_{B}}{\mu_{0} q^{2}+\kappa_{0} q^{4}}$, and the quartic terms yield the desired thermal correction to $\langle h h\rangle_{0}$ via Wick's theorem. To shorten the formulas, in the rest of this paragraph we will drop all factors of $A_{B}$, or set $A_{B}=1$. Retaining only those terms contributing to $r$ we get

$$
\begin{aligned}
\langle h(u) h(0)\rangle_{\text {naive }}= & \langle h(u) h(0)\rangle_{0} \\
& +\frac{\mu_{0}}{8 T}\left\{4 \int \mathrm{d}^{2} u^{\prime}\left\langle h(u) \partial_{a} h\left(u^{\prime}\right)\right\rangle_{0}\left\langle h(0) \partial_{a} h\left(u^{\prime}\right)\right\rangle_{0}\left\langle\partial_{b} h\left(u^{\prime}\right) \partial_{b} h\left(u^{\prime}\right)\right\rangle_{0}\right. \\
& \left.+8 \int \mathrm{d}^{2} u^{\prime}\left\langle h(u) \partial_{a} h\left(u^{\prime}\right)\right\rangle_{0}\left\langle h(0) \partial_{b} h\left(u^{\prime}\right)\right\rangle_{0}\left\langle\partial_{a} h\left(u^{\prime}\right) \partial_{b} h\left(u^{\prime}\right)\right\rangle_{0}\right\} \\
& +\frac{\kappa_{0}}{4 T} 2 \int \mathrm{d}^{2} u^{\prime}\left\langle h(u) \partial_{a} h\left(u^{\prime}\right)\right\rangle_{0}\left\langle h(0) \partial_{a} h\left(u^{\prime}\right)\right\rangle_{0}\left\langle\partial^{2} h\left(u^{\prime}\right) \partial^{2} h\left(u^{\prime}\right)\right\rangle_{0} \\
& +\frac{\kappa_{0}}{T} 2 \int \mathrm{d}^{2} u^{\prime}\left\langle h(u) \partial_{a} h\left(u^{\prime}\right)\right\rangle_{0}\left\langle h(0) \partial_{b} h\left(u^{\prime}\right)\right\rangle_{0}\left\langle\partial_{a} \partial_{b} h\left(u^{\prime}\right) \partial^{2} h\left(u^{\prime}\right)\right\rangle_{0}
\end{aligned}
$$

or

$$
\begin{aligned}
\langle h(q) h(-q)\rangle_{\text {naive }} & =\frac{T}{\kappa_{0} q^{4}+\mu_{0} q^{2}}\left[1+\frac{T}{\kappa_{0} q^{4}+\mu_{0} q^{2}} \int \frac{\mathrm{d}^{2} q^{\prime}}{(2 \pi)^{2}} \frac{\frac{3}{2} \kappa_{0}\left(q^{\prime}\right)^{4}+\mu_{0}\left(q^{\prime}\right)^{2}}{\kappa_{0}\left(q^{\prime}\right)^{4}+\mu_{0}\left(q^{\prime}\right)^{2}}\right] \\
& =T\left\{\mu_{0} q^{2}-\frac{T q^{2}}{2} \int \frac{\mathrm{d}^{2} q^{\prime}}{(2 \pi)^{2}}\left(3-\frac{\mu_{0}\left(q^{\prime}\right)^{2}}{\kappa_{0}\left(q^{\prime}\right)^{4}+\mu_{0}\left(q^{\prime}\right)^{2}}\right)+\mathcal{O}\left(q^{4}\right)\right\}^{-1}
\end{aligned}
$$

so

$$
r_{\text {naive }}=\mu_{0}-\frac{T}{2} \int^{\Lambda} \frac{\mathrm{d}^{2} q}{(2 \pi)^{2}}\left(3-\frac{\mu_{0} q^{2}}{\kappa_{0} q^{4}+\mu_{0} q^{2}}\right) .
$$

Eqn. (2.15) is essentially the result of Meunier [13]. It certainly does not equal (1.2), notwithstanding our formal argument that $r=\tau$.

\section{Gauge Fixing}

Apparently our troubles stem from (2.13). How should we sum over all configurations? We will return in the next section to the question of the spacing of grid points, but even with this choice made correctly, eqn. (2.13) is not correct. Consider the two surfaces $\mathbf{R}$, 
$\mathbf{R}+\mathrm{d} \mathbf{R}$ in Fig. 1. According to (2.4), we describe the displacement from $\mathbf{R}$ to $\mathbf{R}+\mathrm{d} \mathbf{R}$ by an increment $\delta h(u)$ in $h(u)$. But this displacement is only partly physical, i.e., only partly normal to $\mathbf{R}$, since it is along $\mathbf{n}_{0}(u)$, which is not equal to the normal $\mathbf{n}(u)$ at $\mathbf{R}(u)$. The actual normal displacement from $\mathbf{R}$ to $\mathbf{R}+\mathrm{d} \mathbf{R}$ is simply $\mathbf{n}(u) \cdot \mathbf{n}_{0}(u) \delta h(u)$, and we need to replace $[D h]_{\text {naive }}$ in $(2.13)$ by $\mathcal{J}[h][D h]_{\text {naive }}$ where

$$
\mathcal{J}[h] \equiv \prod_{i j}\left(\mathbf{n}\left(u_{i j}\right) \cdot \mathbf{n}_{0}\left(u_{i j}\right)\right) .
$$

The Jacobian $\mathcal{J}[h]$ is the first correction factor (the "Faddeev-Popov determinant") mentioned in the Introduction.

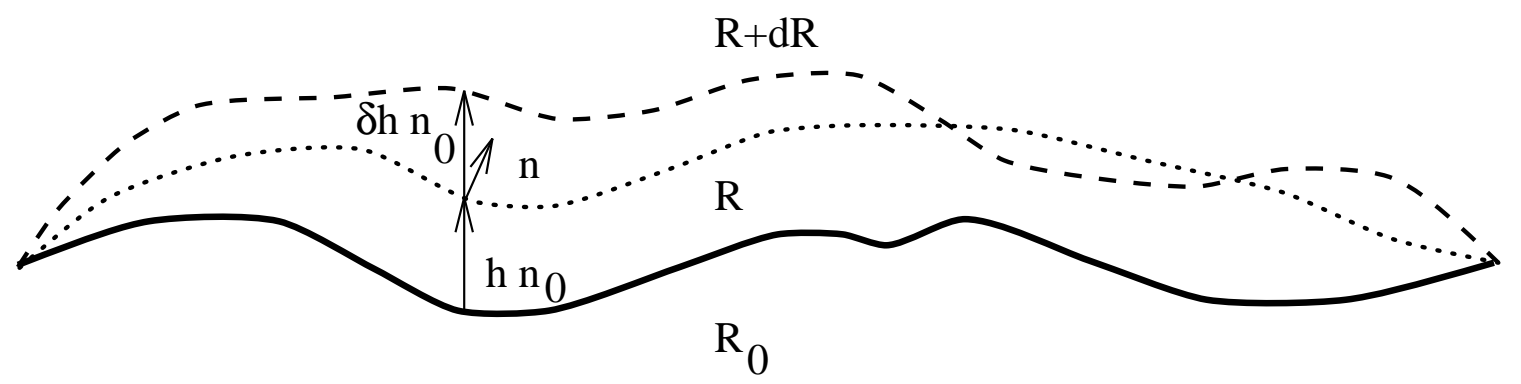

Fig. 1: Two surfaces $\mathbf{R}, \mathbf{R}+\mathrm{d} \mathbf{R}$ described in the normal gauge associated to $\mathbf{R}_{0}$.

Our derivation of (3.1) was heuristic. Nevertheless the result agrees with a more formal derivation which we will give in a moment, and the heuristic discussion gives $\mathcal{J}[h]$ a very simple geometric meaning. The formula (3.1) also agrees with the approach based on metrics on field space [3]. For example, taking $\mathbf{R}_{0}(u)$ to be flat we have $\mathbf{n} \cdot \mathbf{n}_{0}=g^{-1 / 2}$, and $\mathcal{J}$ is the Monge gauge factor found in [3]. It is not unity, contrary to ref. [14]; while it does not affect the one-loop renormalization of $\kappa_{0}$, it will be crucial for $r$, as shown below and in [3].

Our second derivation of (3.1) is couched in language familiar from gauge theories [20]. It is more precise than the motivation just given for eqn. (3.1), and again reproduces the answer given in [3]. We think that each derivation sheds light on (and increases our confidence in) the final answer. In order to form a statistical sum over distinct surfaces we imagine integrating over all parameterized surfaces, with a correction factor to eliminate overcounting. Thus, we write the partition function as

$$
Z=\int[D \mathbf{R}]\left(\frac{1}{\operatorname{vol}[\mathbf{R}]}\right) \mathrm{e}^{-\mathcal{H} / T}
$$


Here $[D \mathbf{R}]$ just describes the possible displacement of the individual mass-points on the surface and $\operatorname{vol}[\mathbf{R}]$ is a factor to be discussed below. All we will need to know for the moment is that the measure $[D \mathbf{R}]$ must be coordinate-invariant, since no preferred coordinate system is given on a fluid membrane; $[D \mathbf{R}]$ must also be invariant under spatial translations and rotations.

To finish specifying (3.2) we need to specify the extra volume factor vol[R] appearing there. Starting from any surface $\mathbf{R}(u)$ and applying all possible reparameterizations we sweep out a subspace of all parameterized surfaces. The volume vol[R] of this "orbit" may well depend on which surface we start with, but it cannot depend on how $\mathbf{R}(u)$ is parameterized.

Since it proves inconvenient to include $\operatorname{vol}[\mathbf{R}]$ explicitly in (3.2), we now fix a coordinate choice by introducing two constraints on $\mathbf{R}(u)$ at every point $u$ :

$$
f_{a}(\mathbf{R}(u))=0 \quad a=1,2
$$

For example, in normal gauge we require

$$
f_{a}(\mathbf{R}(u)) \equiv \lambda^{-2} \mathbf{e}_{a, 0}(u) \cdot\left(\mathbf{R}(u)-\mathbf{R}_{0}(u)\right)=0 \quad .
$$

We have introduced a factor of $\lambda^{-2}$ (see (1.3)) to make $f_{a}$ dimensionless. Any length scale will do here since our answers will be altogether independent of our choice of $f_{a}$. We now define the Faddeev-Popov determinant $\Delta_{f}[\mathbf{R}]$ via

$$
1 \equiv \Delta_{f}[\mathbf{R}] \cdot \int^{\prime}[D \widetilde{\mathbf{R}}] \delta\left[f_{a}(\widetilde{\mathbf{R}})\right]
$$

The integral is only over the space of $\widetilde{\mathbf{R}}$ 's which are reparameterizations of $\mathbf{R}$; hence we also have

$$
\operatorname{vol}[\mathbf{R}]=\int^{\prime}[D \widetilde{\mathbf{R}}]
$$

To keep vol[R], and hence (3.2), dimensionless we include two factors of $\lambda^{-1}$ at every point in (3.5). Since every $\widetilde{\mathbf{R}}(u)=\mathbf{R}(\Omega(u))$ is related to $\mathbf{R}$ by a reparameterization, $u^{a} \mapsto \Omega^{a}(u)$, we can also write $\int^{\prime}[D \widetilde{\mathbf{R}}]$ as $\int[D \Omega] \mathcal{D}[\Omega, \mathbf{R}]$, where formally $\mathcal{D}[\Omega, \mathbf{R}]=\operatorname{det} \frac{\mathrm{d}(\mathbf{R}(\Omega))}{\mathrm{d} \Omega}$ is a Jacobian factor related to the change of variables. Let us now insert (3.4) into (3.1) to get

$$
Z=\int[D \mathbf{R}]\left(\frac{1}{\operatorname{vol}[\mathbf{R}]}\right) \mathrm{e}^{-\mathcal{H}[\mathbf{R}] / T} \Delta_{f}[\mathbf{R}] \int[D \Omega] \mathcal{D}[\Omega, \mathbf{R}] \delta\left[f_{a}(\mathbf{R}(\Omega))\right]
$$


Since a reparameterization $\Omega$ has meaning independent of the surface $\mathbf{R}$ we apply it to, we can do the $\Omega$ integral last. Letting $\widetilde{\mathbf{R}}=\mathbf{R}(\Omega)$, we use the fact that $[D \mathbf{R}], \mathcal{H}$, $\operatorname{vol}[\mathbf{R}]$ and $\Delta_{f}$ are reparameterization invariant to rewrite $Z$ as

$$
Z=\int[D \Omega] \int[D \widetilde{\mathbf{R}}]\left(\frac{1}{\operatorname{vol}[\widetilde{\mathbf{R}}]}\right) \mathrm{e}^{-\mathcal{H}[\widetilde{\mathbf{R}}] / T} \Delta_{f}[\widetilde{\mathbf{R}}] \delta\left[f_{a}(\widetilde{\mathbf{R}})\right] \mathcal{D}[\Omega, \mathbf{R}]
$$

Also, the fact that $[D \mathbf{R}]$ is coordinate-invariant means that small displacements of $\Omega$ sweep out equal volumes no matter where on a given orbit they act, i.e. $\mathcal{D}[\Omega, \mathbf{R}]=\mathcal{D}[\Omega, \widetilde{\mathbf{R}}]$. Renaming $\widetilde{\mathbf{R}}$ as $\mathbf{R}$ and using (3.5) we thus get

$$
Z=\int[D \mathbf{R}] \mathrm{e}^{-\mathcal{H}[\mathbf{R}] / T} \Delta_{f}[\mathbf{R}] \delta\left[f_{a}(\mathbf{R})\right]
$$

The only configurations entering (3.6) are those obeying the gauge condition, as desired, but now we must compute $\Delta_{f}[\mathbf{R}]$ from the definition (3.4).

While (3.6) is quite general, we will now specialize to the normal gauge (2.4) associated to a surface $R_{0}$. The functional delta-function in (3.6) tells us we need only to examine $\Delta_{f}[\mathbf{R}]$ for $\mathbf{R}$ obeying the normal-gauge condition; the delta-function in (3.4) then tells us we need only study $\widetilde{\mathbf{R}}$ infinitesimally close to $\mathbf{R}$. Thus we write $\widetilde{\mathbf{R}}$ in the form (2.3), $\widetilde{\mathbf{R}}(u)=\mathbf{R}(u)-\varepsilon^{a}(u) \mathbf{e}_{a}(u)$. A reasonable choice ${ }^{4}$ for $[D \widetilde{\mathbf{R}}]$ near a given $\mathbf{R}$ is then

$$
[D \widetilde{\mathbf{R}}] \equiv \prod_{i j}\left(\mathrm{~d}^{3} \widetilde{\mathbf{R}}\left(u_{i j}\right) / \lambda^{3}\right)
$$

In (3.7) we imagine that whatever coordinate system we choose, a grid has been laid down with each point carrying constant mass. Since each constituent molecule occupies a fixed physical area, we can equivalently specify that each grid cell of size $\left(\Delta u^{1}, \Delta u^{2}\right)$ occupy equal areas in 3-space. (This, in turn, typically means that the $\Delta u^{a}$ themselves are not all the same.) With this understanding $[D \widetilde{\mathbf{R}}]$ is indeed coordinate-invariant.

Submitting $\mathbf{R}$ to an infinitesimal coordinate transformation $u^{a} \mapsto u^{a}+\varepsilon^{a}(u)$, we see that at each $u_{i j} \mathbf{R}\left(u_{i j}\right)$ moves in the tangent plane; the measure $\mathrm{d}^{3} \widetilde{\mathbf{R}}\left(u_{i j}\right) / \lambda^{3}$ in $(3.7)$, restricted to this plane, becomes

$$
\lambda^{-2}\left|\mathbf{e}_{1} \times \mathbf{e}_{2}\right| \mathrm{d}^{2} \varepsilon\left(u_{i j}\right)=\lambda^{-2} \sqrt{g\left(u_{i j}\right)} \mathrm{d}^{2} \varepsilon\left(u_{i j}\right)
$$

4 The nonlinear measure correction to be discussed in the next section will not affect our answer; see the Appendix. 
We can now evaluate (3.4):

$$
\begin{aligned}
\Delta_{f}[\mathbf{R}]^{-1} & =\int \prod_{i j}\left(\lambda^{-2} \sqrt{g} \mathrm{~d}^{2} \varepsilon\left(u_{i j}\right)\right) \delta^{(2)}\left(f_{a}\left(\mathbf{R}\left(u_{i j}^{a}+\varepsilon^{a}\left(u_{i j}\right)\right)\right)\right) \\
& =\int \prod_{i j}\left(\lambda^{-2} \sqrt{g} \mathrm{~d}^{2} \varepsilon\left(u_{i j}\right)\right) \operatorname{det}^{-1}\left[J_{a b}\left(u_{i j}\right)\right] \delta^{(2)}\left(\varepsilon^{a}\left(u_{i j}\right)\right),
\end{aligned}
$$

where $J_{a b}(u)=\partial f_{a}(\mathbf{R}(u+\varepsilon)) / \partial \varepsilon^{b}=\lambda^{2} \mathbf{e}_{a, 0} \cdot \mathbf{e}_{b}$ in normal gauge using (3.3). Thus

$$
\Delta_{f}[\mathbf{R}]=\prod_{i j} \frac{\operatorname{det} \mathbf{e}_{a}^{0} \cdot \mathbf{e}_{b}}{\sqrt{g}}
$$

To finish our job of getting an explicit expression for the partition function, we now express $[D \mathbf{R}] \delta\left[f_{a}(\mathbf{R})\right]$ appearing in (3.6) in terms of the nonredundant height variable $h(u)$ appearing in (2.4). We can conveniently express an arbitrary $\mathbf{R}$ in terms of a height change plus the rest:

$$
\mathbf{R}(u)=\mathbf{R}_{0}(u)+h(u) \mathbf{n}_{0}(u)+\mathbf{e}_{a, 0}(u) v^{a}(u)
$$

The calculation is not quite the same as the one leading to (3.8); instead of infinitesimal reparameterizations $\varepsilon^{a}$ from an arbitrary normal-gauge surface $\mathbf{R}$, now we have displacements from the fixed reference surface $\mathbf{R}_{0}$. Accordingly, instead of (3.8) we find

$$
\mathrm{d}^{3} \mathbf{R}(u)=\sqrt{g_{0}(u)} \mathrm{d}^{2} v \mathrm{~d} h
$$

Next, (2.4) with (3.10) gives $f_{a}(\mathbf{R})=\lambda^{-2} \mathbf{e}_{a, 0} \cdot \mathbf{e}_{b, 0} v^{b}$, or with $(3.10) \delta^{(2)}\left(f_{a}(\mathbf{R})\right)=$ $\lambda^{2} \operatorname{det}^{-1}\left(\mathbf{e}_{a, 0} \cdot \mathbf{e}_{b, 0}\right) \cdot \delta^{(2)}\left(v^{a}\right)$. We can now do the $v^{a}$ integrals to get from (3.6) to

$$
Z=\int\left[\prod_{i j} \lambda^{-1} d h\left(u_{i j}\right)\right] \widetilde{\Delta} \mathrm{e}^{-\mathcal{H} / T}
$$

where

$$
\widetilde{\Delta}=\prod_{i j} \frac{\operatorname{det}\left(\mathbf{e}_{a, 0} \cdot \mathbf{e}_{b}\right)}{\sqrt{g} \sqrt{g_{0}}}=\prod_{i j} \mathbf{n} \cdot \mathbf{n}_{0}\left(u_{i j}\right)
$$

It is understood that $\mathcal{H}$ and $\widetilde{\Delta}$ are evaluated on the surface given in terms of $h(u)$ by (2.4). The last step follows because $\operatorname{det}\left(\mathbf{e}_{a, 0} \cdot \mathbf{e}_{b}\right)=\left(\mathbf{e}_{1,0} \times \mathbf{e}_{2,0}\right) \cdot\left(\mathbf{e}_{1} \times \mathbf{e}_{2}\right)=\sqrt{g_{0}} \mathbf{n}_{0} \cdot \sqrt{g} \mathbf{n}$. Note that each factor in (3.12) is explicitly coordinate-invariant, in contrast with the analogous formula in [14]. 
Eqns. (3.12)-(3.13) are our final form for the partition function. We see that (3.13) indeed does agree with our heuristic formula (3.1). Specializing to Monge gauge, $\mathbf{n}_{0}=\hat{z}$, we recover the result of [3], $\widetilde{\Delta}=\prod_{i j} g^{-1 / 2}$.

An alternate form for $\widetilde{\Delta}$ can be obtained using

$$
\mathbf{e}_{b}=\partial_{b} \mathbf{R}=\mathbf{e}_{b, 0}+\partial_{b}\left(h \mathbf{n}_{0}\right)=\mathbf{e}_{b, 0}-h \mathbf{e}_{0}^{c} K_{b c, 0}+\partial_{b} h \mathbf{n}_{0}
$$

where $K_{b c, 0}$ is the curvature tensor of the background surface. From this we find

$$
\widetilde{\Delta}=\prod_{i j} \frac{\operatorname{det}\left(g_{a b, 0}-h K_{a b, 0}\right)}{\sqrt{g_{0}} \sqrt{g}}=\exp \sum_{i j}\left(-\frac{1}{2} \partial^{a} h \partial_{a} h+\mathcal{O}\left(h^{4}\right)\right)
$$

which again differs slightly from the formula in [14]. We see that getting the correct $\sqrt{g}$ factors in the denominator is crucial to avoid a spurious term linear in $h$.

Returning to the unhappy ending of sect. 3, we now see that the Monge-gauge FaddeevPopov factor

$$
\widetilde{\Delta}=\exp \left[-\frac{1}{T} \sum_{i j} \frac{T}{2} \log \left(1+(\partial h)^{2}\right)\right]=\exp \left[-\frac{1}{T} \sum_{i j} \frac{T}{2}(\partial h)^{2}\right]
$$

effectively contributes a new term to (2.12), one which is already of order $T$. Following the steps leading to (2.15), we get the corrected version

$$
r_{\mathrm{FP}}=\mu_{0}-\frac{T}{2} \int^{\Lambda} \frac{\mathrm{d}^{2} q}{(2 \pi)^{2}}\left(1-\frac{\mu_{0} q^{2}}{\kappa_{0} q^{4}+\mu_{0} q^{2}}\right) .
$$

On the other hand $\widetilde{\Delta}$ cannot contribute to our calculation of $\tau$ since being already of order $T$ we simply evaluate it at $h=0$, where it equals one. We still have the problem that $r_{\mathrm{FP}} \neq \tau$.

\section{Nonlinear Measure}

To see where we have erred, let us first give another derivation that $r=\tau$. Note that there are many different Monge gauges corresponding to projections to various reference planes. ${ }^{5}$ So far we have used a reference plane coinciding with the plane of the frame

5 Recall that a Monge gauge is a normal gauge where the reference surface $\mathbf{R}_{0}$ in Fig. 1 is flat. Alternatively we could imagine two different physical surfaces related by a rotation, both viewed in the same Monge gauge. 
holding the membrane, so the equilibrium configuration is $\bar{h}=0$, but we could instead choose $\mathbf{R}_{0}^{\prime}$ tilted by a small angle $\psi$. We will always work to lowest nontrivial order in $\psi$. In the new coordinate system the same equilibrium surface is now described by $\bar{h}^{\prime}(u)=\psi u^{1}$ (Fig. 2); furthermore the projected area has been foreshortened in one direction, giving $A_{B}^{\prime}=A_{B} \cos \psi=A_{B}\left(1-\frac{1}{2} \psi^{2}\right)$. Since the two calculations compute the same quantity we must have that

$$
\begin{aligned}
\Gamma\left[A_{B} ; \bar{h}=0\right] & \equiv \tau A_{B} \\
& =\Gamma\left[A_{B}-\frac{1}{2} \psi^{2} A_{B} ; \bar{h}^{\prime}(u)=\psi u^{1}\right] \\
& =\tau A_{B}-\tau \frac{1}{2} \psi^{2} A_{B}+r \frac{1}{2} \psi^{2} A_{B} .
\end{aligned}
$$

In the last step we wrote the $\mathcal{O}\left(\bar{h}^{\prime 2}\right)$ term of $\Gamma$ in terms of $r\left(\partial \bar{h}^{\prime}\right)^{2}=r \psi^{2}$. Thus we again conclude $r=\tau$, contrary to our still incomplete calculation.

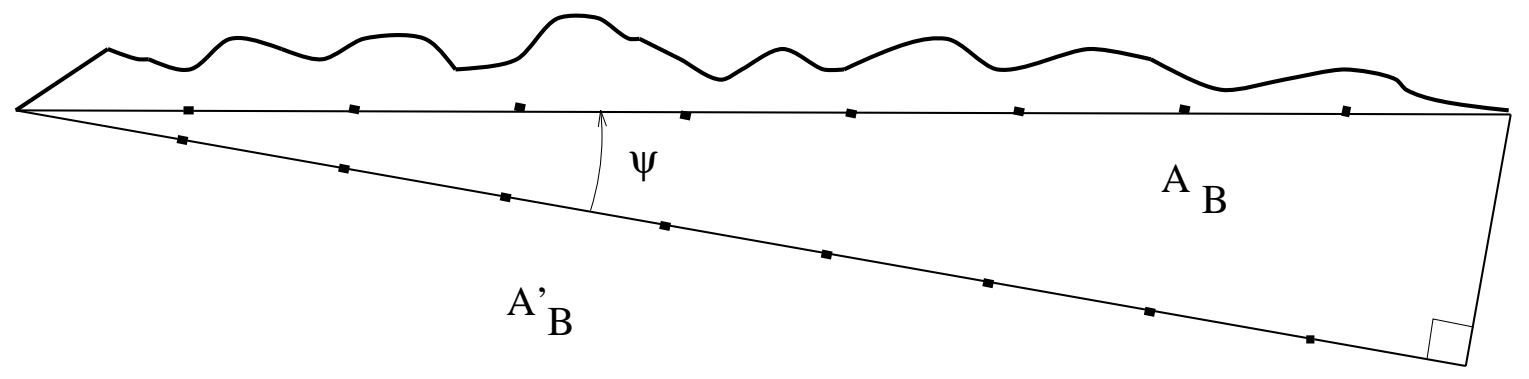

Fig. 2: Tilting the reference surface foreshortens both the base area and the coordinate cutoff.

What have we done wrong? Throughout our discussion of the previous section we assumed that the measure $[D \mathbf{R}]$, and its reduction $[D h]$, were invariant under changes of parameterization of the surface. But the choice made in (2.13) does not have this property. We chose the grid points $\left\{u_{i j}\right\}$ evenly spaced in the coordinates $u^{a}$, which has different meaning for different coordinate systems. In particular for our second description we have a total of $A_{B}^{\prime} / a^{2}$ degrees of freedom, which is different from the true number $A_{B} / a^{2}$ of mass points! More formally, eqn. (3.7) tells us to use grid cells of constant metric area $\Delta u^{1} \Delta u^{2}=a^{2} / \sqrt{g}$, while (2.13) specifies constant coordinate area $\Delta u^{1} \Delta u^{2}=a^{2}$. We can easily show that this error accounts for the remaining discrepancy between $\tau$ and $r_{\mathrm{FP}}$, eqns. (1.2) and (3.17).

Let us denote by $\Gamma_{\mathrm{FP}}\left[A_{B}, \Lambda^{1}, \Lambda^{2} ; \bar{h}\right]$ the effective action calculated with the correct Faddeev-Popov factor $\mathcal{J}$ but still using the naive (coordinate-dependent) measure (2.13). We momentarily allow an anisotropic cutoff $\left(\Lambda^{1}, \Lambda^{2}\right)$, which we write explicitly; at the end of the discussion we will set $\Lambda^{1}=\Lambda^{2}=2 \pi / a$. The foregoing discussion implies that we 
may use $\Gamma_{\mathrm{FP}}$ if we remember to foreshorten the spacing of constituents (increase $\Lambda^{1}$ ) as well as the base plane (Fig. 2), so (4.1) becomes

$$
\begin{aligned}
\Gamma_{\mathrm{FP}}\left[A_{B}, \frac{2 \pi}{a}, \frac{2 \pi}{a}, \bar{h}=0\right] & =\tau A_{B} \\
& =\Gamma_{\mathrm{FP}}\left[A_{B}-\frac{1}{2} \psi^{2} A_{B},\left(1+\frac{1}{2} \psi^{2}\right) \frac{2 \pi}{a}, \frac{2 \pi}{a} ; \bar{h}^{\prime}(u)=\psi u^{1}\right]
\end{aligned}
$$

or

$$
\tau A_{B}=\tau A_{B}-\frac{1}{2} \tau \psi^{2} A_{B}+\frac{1}{2} r_{\mathrm{FP}} \psi^{2} A_{B}+\left.\frac{1}{2} \psi^{2} \frac{2 \pi}{a} \frac{\partial \Gamma_{\mathrm{FP}}\left[A_{B}, \Lambda^{1}, \Lambda^{2}, \bar{h}=0\right]}{\partial \Lambda^{1}}\right|_{\Lambda^{1}=\Lambda^{2}=2 \pi / a}
$$

since $r_{\mathrm{FP}}$ is the $q^{2}$ coefficient we calculated with the naive measure. Thus we find

$$
\tau=r_{\mathrm{FP}}+\frac{\partial \tau}{\partial \log \Lambda^{1}}
$$

which really is obeyed by our calculated expressions (1.2), (3.17).

We have thus identified the problem: the naive measure spreads mass points evenly in coordinate space, not in real space. For flat equilibrium surfaces ( $h$ linear in $u^{a}$ ), the right prescription was easy to find: we keep the grid points uniform but change their density to get the RHS of (4.2). Clearly for nonflat $h(u)$ we will have to be more clever, since here the cutoff is nonuniform and indeed field-dependent: the desired measure $[D h]$ is nonlinear in $h$. Incidentally, the same problem we face here arises again in the dynamics context [5], where a naive calculation gives the two-point function controlled by our $r_{\mathrm{FP}}$.

We hinted at the answer in the Introduction: a change in the cutoff, even a spatiallyvarying change, amounts to a local change in the number of degrees of freedom. We can imagine passing from the desired nonlinear $[D h]$ to the convenient $[D h]_{\text {naive }}$ by a sort of decimation procedure, where we integrate $\mathrm{e}^{-\mathcal{H} / T}$ over the discrepant degrees of freedom to obtain $\mathrm{e}^{-\mathcal{H}_{\text {eff }} / T}$. The difference $\delta \mathcal{H}=\mathcal{H}_{\text {eff }}-\mathcal{H}$ is simply a counterterm; while in general it is a nonlocal functional of $h$, still when we ask long-scale questions it may be replaced by a renormalizable truncation, just as we did for $\mathcal{H}$ itself.

We therefore seek a correction factor $\delta \mathcal{H}$ with the property that

$$
\text { " }[D h]=\mathrm{e}^{-\delta \mathcal{H}[h] / T}[D h]_{\text {naive }} "
$$

This equation is in quotes because as it stands it makes no sense: we cannot equate two measures with different numbers of degrees of freedom. In particular $\delta \mathcal{H}$ is not a 
Jacobian factor, as often asserted ${ }^{6}$, but rather is a counterterm. As such it can and will depend on $\mathcal{H}$ itself. Eqn. (4.5) is not a change of variables but rather an assertion about renormalization, namely that each side when weighted by $\mathrm{e}^{-\mathcal{H} / T}$ yields identical moments of $h$, at least for long wavelengths. The usual miracle of renormalization is that adjusting just a few coefficients in $\delta \mathcal{H}$ suffices to make all the moments agree.

What is $\delta \mathcal{H}$ ? We have argued that it is renormalizable. Since it arises from fluctuations it will be explicitly of order $T$, like the Faddeev-Popov correction, and so we will only need its form to $\mathcal{O}\left(h^{2}\right)$ in order to calculate $\langle h h\rangle$ to one loop. (In the Appendix we show how to do better than this.) Dimension counting says it can have at most four derivatives; translation invariance requires at least two. Furthermore $\delta \mathcal{H}$ should depend on $h$ only via the induced metric $g=1+(\partial h)^{2}$, since it reflects a change of cutoff controlled by this metric, and in particular $\delta \mathcal{H}$ should vanish at $h=0$, since here $g_{a b}=\delta_{a b}$ is the metric assumed in $[D h]_{\text {naive }}$. Putting all these arguments together we can only have

$$
\delta \mathcal{H}=\frac{1}{2} T \mu_{1} \int \mathrm{d}^{2} u(\partial h)^{2}+\mathcal{O}\left(h^{4}\right)
$$

where $\mu_{1}$ is some cutoff-dependent constant we are to find. (The reader who is uncomfortable with the logic of this paragraph will find in the Appendix a more deductive derivation of $\delta \mathcal{H}$ obtained by matching two calculations of $\Gamma[\bar{h}]$.)

We remark that our $\delta \mathcal{H}$ is analogous to the "Liouville correction factor" familiar from the string-theory literature [17][18]. In these papers the authors argue in analogy with the conformal anomaly [21] that a covariantly cut-off measure (typically the measure for the scale factor $\mathrm{e}^{\sigma}$ of an internal metric) "equals" a measure cut off by a fixed metric $\left(g_{0}\right)_{a b}$ times a correction:

$$
\text { " }[D \sigma]=\mathrm{e}^{\delta \mathcal{H}[\sigma] / T}[D \sigma]_{g_{0}} ",
$$

where

$$
\delta \mathcal{H}[\sigma]=T \int \sqrt{g_{0}} \mathrm{~d}^{2} u\left[\alpha\left((\partial \sigma)^{2}+R_{0} \sigma\right)+\mu_{1} \mathrm{e}^{\sigma}\right]
$$

and $R_{0}$ is the gaussian curvature of $g_{0}$. We are not using an internal metric, but our (4.6) is reminiscent of the last term of (4.7) (plus a constant).

The pleasant feature of our approach is that fixing the coefficient $\mu_{1}$ by comparison to a simple situation then lets us compute $\Gamma[\bar{h}]$ in any situation. The simple situation we have in mind is the tilted but flat reference surface appearing on the RHS of $(4.2) ; \delta \mathcal{H}\left[\bar{h}=\psi u^{1}\right]$

6 We thank J. Distler for emphasizing this point. 
is supposed to reproduce the effect of cutoff-stretching, which we introduced by hand via the last term of (4.3). Thus we choose $\mu_{1}=\frac{\partial \tau}{\partial \log \Lambda^{1}}$, or

$$
\mu_{1}=\frac{T}{2} \int^{\Lambda} \frac{\mathrm{d}^{2} q}{(2 \pi)^{2}}\left[\log \left(\mu_{0} q^{2}+\kappa_{0} q^{4}\right) \frac{(a \lambda)^{2}}{2 \pi T}+1-\frac{\mu_{0} q^{2}}{\kappa_{0} q^{4}+\mu_{0} q^{2}}\right]
$$

Eqn. (4.8) fixes the effective Hamiltonian to order $h^{2}$. Since (4.6) is effectively just an additional contribution to the $q^{2}$ coefficient of $\langle h h\rangle$, we see that indeed the choice (4.8) required by rotational invariance is also the choice which secures $r=\tau$. To calculate four-point correlators, or two-loop corrections to the effective couplings $\mu_{\text {eff }}, \kappa_{\text {eff }}$ we would need also the $\mathcal{O}\left(h^{4}\right)$ terms of (4.6); see the Appendix.

\section{Conclusion}

If we have explained ourselves clearly the reader may find our analysis somewhat tautological. After all, we uncovered a paradox, only to eliminate it with a mysterious counterterm chosen solely for that purpose! Let us now comment on the real content of our analysis.

We have studied the construction of statistical ensembles of random surfaces made out of fixed-size constituents. Such ensembles are good models for the long-scale behavior of fluid bilayer membranes, though it is straightforward to introduce in-plane order as well. The correct statistical weight for such an ensemble involves a subtle nonlinear measure $[D h]$ not directly suited to diagrammatic perturbation theory. We have argued that this measure may be replaced by a simple one if we incorporate two correction factors into an effective Hamiltonian.

We have given simple geometrical interpretations to the two correction factors, as follows: the Faddeev-Popov term describes how a change $\delta h$ of the normal displacement may not itself be a normal displacement (Fig. 1). The Liouville term describes how the molecules of a surface, projected down to a reference plane, seem to crowd together in regions where the surface is tilted relative to that plane (Fig. 2). These simple pictures, plus some general arguments, enabled us to write down the complete form of the effective Hamiltonian up to terms of order $T$. This accuracy sufficed to show these corrections are crucial to secure the covariant form of the effective action argued on general grounds in sect. 2. Besides being satisfying, the geometrical arguments made it easier to get the right answers than other more formal arguments. 
Our argument feels tautological because we used one consistency condition $(r=\tau)$ to fix one counterterm $\left(\mu_{1}\right)$. In the appendix we sketch how to fix the rest of $\delta \mathcal{H}$ to one loop accuracy, but to go farther we would need to fix its form in advance by imposing some sort of Ward identity, perhaps along the lines of [18]. This task we leave for future work.

\section{Acknowledgements}

We would like to thank J. Polchinski, A.M. Polyakov, and especially J. Distler for conceptual help. P.N. thanks the Aspen Center for Physics for hospitality while some of this work was done. This work was supported in part by NSF grants PHY88-57200, DMR-91-20668 and DMR-91-22645, and the Donors of the Petroleum Research Fund.

\section{Appendix A. Some Calculations}

In Section 4 we used rotational invariance to fix the value of part of the cutoffstretching factor; here we present another approach. Our strategy is to compute the effective action in two different ways; demanding that the results of these calculations agree determines the cutoff-stretching factor to one-loop accuracy, i.e. leading nontrivial order in $T$.

We follow the standard procedure for computing the one-loop effective action, $\Gamma[\bar{h}]$ [21]. We write $h$ as $\bar{h}+\delta h$, expand the energy functional to quadratic order in $\delta h$, and drop the term linear in $\delta h$. Then we do the gaussian integral over $\delta h$ to get the one-loop contribution $\delta \Gamma[\bar{h}]$ to the effective action. Our key observation is that for gaussian integrals we don't really need a measure on field space, since in the end we just compute functional determinants, and for this we need only a metric on fluctuations $\delta h$ about the given $\bar{h}$. Equivalently, to one-loop accuracy we make no error if we replace the full measure $[D \delta h]$, which is nonlinear in $\delta h$, by the cut off measure

$$
[D \delta h]_{\bar{g}}=\prod_{i j, \bar{g}} \frac{\mathrm{d} \delta h\left(u_{i j}\right)}{\lambda}
$$

where the notation " $i j, \bar{g}$ " means that the grid points are spaced uniformly in terms of the distance given by the background metric $\bar{g}_{a b}=\delta_{a b}+\partial_{a} \bar{h} \partial_{b} \bar{h}$, with density $a^{-2}=(\Lambda / 2 \pi)^{2}$. 
As we emphasize below this does not necessarily mean the points are spaced uniformly in any given coordinate system. The measure (A.1) is independent of $\delta h$, unlike the desired $[D \delta h]$, but both correspond to the same metric on fluctuations about $\bar{h}$.

Stated succinctly, in our first calculation we cut off using $\bar{g}$ and no cutoff-stretching correction (though we do need the Faddeev-Popov factor as usual).

It is a bit unsatisfying to have to use a different measure (A.1) for every background $\bar{h}$. Moreover, while we succeeded in determining the free energy for various backgrounds $\bar{h}$, this is not quite the same as finding the correlations of height; for the latter, the $\delta h$-dependence of the true measure omitted from (A.1) will matter. So in our second calculation we replace $[D \delta h]$ not by $[D \delta h]_{\bar{g}}$ but by

$$
\mathrm{e}^{-\delta \mathcal{H}[h] / T}[D \delta h]_{g_{0}}
$$

Here $[D \delta h]_{g_{0}}$ is defined analogously to (A.1) except that now $g_{0, a b}=\delta_{a b}$ is the usual flat metric, so there is no explicit dependence on the background $\bar{h}$ : we have one measure from which we compute the various $\Gamma[\bar{h}]$. Since $g_{0}$ is flat, in this calculation the grid points really are spaced uniformly in the coordinates $u^{a}$. On the other hand, now we have to expect a correction $\delta \mathcal{H}$ as discussed in the text. We will find $\delta \mathcal{H}$ to one loop by recomputing $\Gamma[\bar{h}]$ and comparing to the first calculation; it turns out that a single universal $\delta \mathcal{H}$ secures agreement with all the different $\Gamma$ 's in the first calculation, which used a different measure for each $\bar{h}$. With $\delta \mathcal{H}$ in hand we can then proceed to the calculation of $\langle h h\rangle$.

We begin our calculations by quoting some Monge gauge formulas. We have the following expressions for the volume element, the inverse metric, and the mean curvature [11]:

$$
\begin{gathered}
\sqrt{g} \mathrm{~d}^{2} u=\sqrt{1+(\partial h)^{2}} \mathrm{~d}^{2} u, \\
g^{a b}=\delta_{a b}-\frac{\partial_{a} h \partial_{b} h}{1+(\partial h)^{2}}, \\
\operatorname{Tr} K=\frac{-\partial^{2} h}{\sqrt{1+(\partial h)^{2}}}+\frac{\partial_{a} \partial_{b} h \partial_{a} h \partial_{b} h}{\left(1+(\partial h)^{2}\right)^{3 / 2}} .
\end{gathered}
$$

The energy functional (2.2) becomes

$$
\begin{aligned}
\mathcal{H}=\frac{\kappa_{0}}{2} \int \mathrm{d}^{2} u & \left\{\frac{\left(\partial^{2} h\right)^{2}}{\sqrt{1+(\partial h)^{2}}}-\frac{2 \partial^{2} h \partial_{a} h \partial_{b} h \partial_{a} \partial_{b} h}{\left(1+(\partial h)^{2}\right)^{3 / 2}}+\frac{\left(\partial_{a} h \partial_{b} h \partial_{a} \partial_{b} h\right)^{2}}{\left(1+(\partial h)^{2}\right)^{5 / 2}}\right\} \\
& +\mu_{0} \int \mathrm{d}^{2} u \sqrt{1+(\partial h)^{2}}
\end{aligned}
$$


We work to all orders in the height.

We are now ready for the first of our two calculations. Following the above program, we replace $h$ by $\bar{h}+\delta h$ and retain the terms quadratic in $\delta h$. In the following we drop all terms that do not contribute to the frame tension renormalization, i.e. terms with more derivatives than $\bar{h}$ 's, though it's not much harder to retain them. We then have the quadratic bit of $\mathcal{H}$

$$
\begin{gathered}
\mathcal{H}^{I I}=\frac{\kappa_{0}}{2} \int \mathrm{d}^{2} u \sqrt{\bar{g}} \delta h\left\{\frac{1}{\bar{g}} \partial^{4}-\frac{2 \partial_{a} \bar{h} \partial_{b} \bar{h}}{\bar{g}^{2}} \partial^{2} \partial_{a} \partial_{b}+\frac{\partial_{a} \bar{h} \partial_{b} \bar{h} \partial_{c} \bar{h} \partial_{d} \bar{h}}{\bar{g}^{3}} \partial_{a} \partial_{b} \partial_{c} \partial_{d}\right\} \delta h \\
+\frac{\mu_{0}}{2} \int \mathrm{d}^{2} u \sqrt{\bar{g}} \delta h\left\{-\frac{1}{\bar{g}} \partial^{2}+\frac{\partial_{a} \bar{h} \partial_{b} \bar{h}}{\bar{g}^{2}} \partial_{a} \partial_{b}\right\} \delta h
\end{gathered}
$$

Note that we have pulled out a factor of $\sqrt{\bar{g}}$ so that upon discretizing this integral (A.7) the grid points are spaced with uniform density according to the metric $\bar{g}_{a b}$ (see (A.1)). The result of this gaussian integration is

$$
\delta \Gamma_{\text {gaussian }}[\bar{h}]=\frac{T}{2} \operatorname{Tr}_{\bar{g}} \log \left(\frac{\kappa_{0} \lambda^{2} a^{2}}{2 T \pi}\right) \frac{1}{\bar{g}}\left[\left(\bar{g}^{a b} \partial_{a} \partial_{b}\right)^{2}+\frac{\mu_{0}}{\kappa_{0}}\left(\bar{g}^{a b} \partial_{a} \partial_{b}\right)\right]
$$

In this equation the symbol " $\operatorname{Tr}_{\bar{g}}$ " means that we evaluate the trace of the following operator using a cutoff equivalent to the point spacing specified in (A.1). In momentum space this means that we find a basis of eigenmodes of the covariant laplacian $\bar{\Delta}=\frac{1}{\sqrt{\bar{g}}} \partial_{a} \bar{g}^{a b} \sqrt{\bar{g}} \partial_{b}$, then sum over all modes with eigenvalues less than $(\Lambda)^{2}$. This prescription is coordinateinvariant; if $\bar{g}_{a b}=\delta_{a b}$ then it just amounts to integrating over a circular Brillouin zone as usual. We have simplified (A.8) by factoring out the constants and one power of $\bar{g}^{-1}$.

Eqn. (A.8) is easy to work with because we can now write $\bar{g}^{a b} \partial_{a} \partial_{b}$ as $\bar{\Delta}$ plus terms with more derivatives than $\bar{h}$ 's, which we are dropping:

$$
\delta \Gamma_{\text {gaussian }}[\bar{h}]=\frac{T}{2} \operatorname{Tr}_{\bar{g}} \log \frac{\kappa_{0}(\lambda a)^{2} \Lambda^{4}}{2 \pi T}-\frac{T}{2} \operatorname{Tr}_{\bar{g}} \log \bar{g}+\frac{T}{2} \operatorname{Tr}_{\bar{g}} \log \left(\bar{\Delta}^{2}-\frac{\mu_{0}}{\kappa_{0}} \bar{\Delta}\right) \Lambda^{-4} .
$$

To this expression we must add the contribution from the Faddeev-Popov factor $\prod_{i j, \bar{g}} \bar{g}^{-1 / 2}$, which we write as $\exp \left[-\frac{1}{T}\left(\frac{1}{2} T \operatorname{Tr}_{\bar{g}} \log \bar{g}\right)\right]$ :

$$
\delta \Gamma[\bar{h}]=\frac{T}{2} \operatorname{Tr}_{\bar{g}} \log \frac{\kappa_{0} \lambda^{2} a^{2} \Lambda^{4}}{2 \pi T}+\frac{T}{2} \operatorname{Tr}_{\bar{g}} \log \bar{g}-\frac{T}{2} \operatorname{Tr}_{\bar{g}} \log \bar{g}+\frac{T}{2} \operatorname{Tr}_{\bar{g}} \log \left(\frac{\bar{\Delta}^{2}}{\Lambda^{4}}-\frac{\mu_{0}}{\kappa_{0} \Lambda^{4}} \bar{\Delta}\right)
$$

Note that the noncovariant Faddeev-Popov factor exactly cancels with the noncovariant factor of $\bar{g}^{-1}$ from the expansion of the energy functional, leaving us with a contribution 
to the effective action which is covariant to all orders in the height $\bar{h}$. It is easy to evaluate the first trace:

$$
\operatorname{Tr}_{\bar{g}} \log \frac{\kappa_{0} \lambda^{2} a^{2} \Lambda^{4}}{2 \pi T}=\log \frac{\kappa_{0} \lambda^{2} a^{2} \Lambda^{4}}{2 \pi T} \sum_{q} 1
$$

The sum is just the total number of mass points $a^{-2} \int \mathrm{d}^{2} u \sqrt{\bar{g}}$, which we will write as

$$
=\int \mathrm{d}^{2} u \sqrt{\bar{g}} \int^{\Lambda} \frac{\mathrm{d}^{2} q}{(2 \pi)^{2}} \log \frac{\kappa_{0} \lambda^{2} a^{2} \Lambda^{4}}{2 \pi T} .
$$

To make the last term of (A.10) tractable we will now specialize to a special class of backgrounds $\bar{h}$ for which we know the eigenmodes of $\bar{\Delta}$ explicitly, namely $\bar{h}(u)$ linear in $u^{a}$. Examining this case suffices to let us extract the coefficients of all the $(\partial \bar{h})^{n}$ terms. $^{7}$ For such a $\bar{g}_{a b}$ we have

$$
\begin{aligned}
& \operatorname{Tr}_{\bar{g}} \log \left(\bar{\Delta}^{2}-\frac{\mu_{0}}{\kappa_{0}} \bar{\Delta}\right) \Lambda^{-4} \\
& \quad=\int \mathrm{d}^{2} u \int_{q_{a} q_{b} \bar{g}^{a b}<\Lambda} \frac{\mathrm{d}^{2} q}{(2 \pi)^{2}} \log \left(\left(q_{a} q_{b} \bar{g}^{a b}\right)^{2}+\frac{\mu_{0}}{\kappa_{0}} q_{a} q_{b} \bar{g}^{a b}\right) \Lambda^{-4} .
\end{aligned}
$$

After a linear transformation of integration variables we get

$$
=\int \mathrm{d}^{2} u \sqrt{\bar{g}} \int_{k^{2}<\Lambda} \frac{\mathrm{d}^{2} k}{(2 \pi)^{2}} \log \left(k^{4}+\frac{\mu_{0}}{\kappa_{0}} k^{2}\right) \Lambda^{-4} .
$$

The final result for the renormalized frame tension is thus

$$
\tau=\mu_{0}+\frac{T}{2} \int^{\Lambda} \frac{\mathrm{d}^{2} q}{(2 \pi)^{2}} \log \frac{\lambda^{2} a^{2}}{2 \pi T}\left(\kappa_{0} q^{4}+\mu_{0} q^{2}\right)
$$

which is indeed the contribution to (1.2) due to the modes in question, and agrees with $[15] .^{8}$

We now turn to our second derivation described above: we compute the part of the effective action involving the renormalized frame tension using the measure cut off by a fixed metric $g_{0, a b}=\delta_{a b}$. To space the grid points with uniform density according to $g_{0, a b}$, we write (A.7) as

$$
\mathcal{H}^{I I}=\frac{\kappa_{0}}{2} \int \mathrm{d}^{2} u \sqrt{g_{0}} \delta h \frac{1}{\sqrt{g_{0} \bar{g}}}\left(\bar{\Delta}^{2}-\frac{\mu_{0}}{\kappa_{0}} \bar{\Delta}\right) \delta h .
$$

7 Alternately we could simplify the sum by considering only the contribution from a thin shell in momentum space.

8 We have corrected an error in the formula following (B.2) in [3]; it had an extra factor of $\sqrt{\bar{g}}$ (cf. (A.13)). 
While the laplacian $\bar{\Delta}$ associated to $\bar{g}$ (not $g_{0}$ ) again appears in (A.15), now our gaussian integral is cut off using a basis of eigenmodes of the flat $\partial^{2}$ :

$$
\delta \widetilde{\Gamma}_{\text {gaussian }}=\frac{T}{2} \operatorname{Tr}_{g_{0}} \log \frac{\kappa_{0} \lambda^{2} a^{2}}{2 \pi T} \frac{1}{\sqrt{g_{0} \bar{g}}}\left(\bar{\Delta}^{2}-\frac{\mu_{0}}{\kappa_{0}} \bar{\Delta}\right)
$$

To (A.9) we must add the Faddeev-Popov factor and the cutoff-stretching factor. Both of these are already $\mathcal{O}(T)$, so to one loop we simply evaluate them at $\bar{h}$ :

$$
\delta \Gamma=\frac{T}{2} \operatorname{Tr}_{g_{0}} \log \frac{\kappa_{0} \lambda^{2} a^{2}}{2 \pi T} \frac{1}{\sqrt{g_{0} \bar{g}}}\left(\bar{\Delta}^{2}-\frac{\mu_{0}}{\kappa_{0}} \bar{\Delta}\right)+\delta \mathcal{H}[\bar{h}]+\frac{T}{2} \operatorname{Tr}_{\bar{g}} \log \bar{g}
$$

It is now easy to see what factor $\mathrm{e}^{-\delta \mathcal{H}[h] / T}$ belongs in (A.2). $\delta \mathcal{H}[h]$ should be the functional of $h$ which, when evaluated at $\bar{h}$, reconciles (A.17) with (A.10):

$$
\delta \mathcal{H}[h]=\frac{T}{2}\left(\operatorname{Tr}_{g}-\operatorname{Tr}_{g_{0}}\right) \log \frac{\kappa_{0} \lambda^{2} a^{2}}{2 \pi T g}\left(\Delta^{2}-\frac{\mu_{0}}{\kappa_{0}} \Delta\right)-\frac{T}{2} \operatorname{Tr}_{g_{0}} \log \left(\frac{g}{g_{0}}\right)^{1 / 2}
$$

We have written the correction factor this way to emphasize how at least part of it (the first term of (A.18)) is clearly a result of decimation. As in (A.13) we can write the first term of (A.18) as the difference of the integral over an elliptical Brillouin zone minus the integral over a smaller, circular, zone with the same density of points $A_{B}$ (Fig. 3). Thus the first term of (A.18) may be written as

$$
\left(\int \mathrm{d}^{2} u \int_{B Z^{\prime}} \frac{\mathrm{d}^{2} q}{(2 \pi)^{2}}-\int \mathrm{d}^{2} u \int_{B Z} \frac{\mathrm{d}^{2} q}{(2 \pi)^{2}}\right) \log \frac{\lambda^{2} a^{2}}{2 \pi T}\left(\kappa_{0}\left(g^{a b} q_{a} q_{b}\right)^{2}+\mu_{0} g^{a b} q_{a} q_{b}\right)
$$

where $q$ is in $B Z^{\prime}$ if $q_{a} q_{b} g^{a b}<\Lambda^{2}$ and $q$ is in $B Z$ if $q^{2}<\Lambda^{2}$.

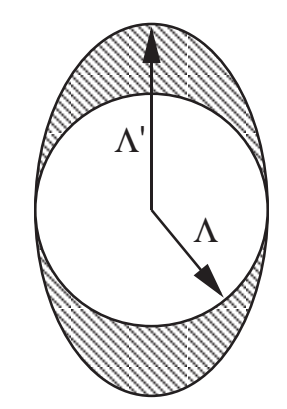

Fig. 3: The origin of the cutoff-stretching counterterm as a difference between two Brillouin zones. The stretched cutoff $\Lambda^{\prime}$ equals $\Lambda / \cos \psi$ if the background surface $\bar{h}$ is a flat plane tilted by an angle $\psi$ relative to the reference plane. 
We can recast (A.18) as follows:

$$
\begin{aligned}
\delta \mathcal{H}[h]= & \frac{T}{2} \int \mathrm{d}^{2} u \sqrt{\bar{g}} \int \frac{\mathrm{d}^{2} q}{(2 \pi)^{2}} \log \frac{\lambda^{2} a^{2}}{2 \pi T}\left(\kappa_{0} q^{4}+\mu_{0} q^{2}\right) \\
& -\frac{T}{2} \int \mathrm{d}^{2} u \int \frac{\mathrm{d}^{2} q}{(2 \pi)^{2}} \log \frac{\lambda^{2} a^{2}}{2 \pi T}\left(\kappa_{0}\left(g^{a b} q_{a} q_{b}\right)^{2}+\mu_{0} g^{a b} q_{a} q_{b}\right) \\
& -\frac{T}{2} \int \mathrm{d}^{2} u \sqrt{\bar{g}} \int \frac{\mathrm{d}^{2} q}{(2 \pi)^{2}} \log \bar{g}
\end{aligned}
$$

In this form we can readily see that the second line contributes a term to the free energy cancelling the contribution from Feynman diagrams, the third line cancels the contribution from the Faddeev-Popov determinant, and the remaining first line is covariant as required. From this form one can show that all the terms of $\delta \mathcal{H}$ proportional to $(\partial h)^{n}$ are just what are needed to satisfy the Ward-like identity (4.1) to all orders in the angle $\psi$.

Finally, we can expand (A.18) in terms of $h$; the first couple of terms are

$$
\begin{aligned}
& \delta \mathcal{H}[h]=\frac{T}{2} \int \mathrm{d}^{2} u \int^{\Lambda} \frac{\mathrm{d}^{2} q}{(2 \pi)^{2}} {\left[\log \frac{\lambda^{2} a^{2}\left(\kappa_{0} q^{4}+\mu_{0} q^{2}\right)}{2 \pi T}+1-\frac{\mu_{0} q^{2}}{\kappa_{0} q^{4}+\mu_{0} q^{2}}\right] \cdot \frac{1}{2}(\partial h)^{2} } \\
&+\mathcal{O}\left(h^{4}\right),
\end{aligned}
$$

in agreement with (4.8).

To conclude our analysis, we finally compute the last correction to the $q^{2}$ coefficient $r$. As mentioned earlier, the $\mathcal{O}\left(h^{2}\right)$ term of (A.21) leads to a new correction to $\langle h h\rangle$, not accounted for in the steps leading to (3.17). Since this term is $\mathcal{O}(T)$, it gives a new tree Feynman graph, which when added to the 1-loop part of (3.17) gives $r=\tau$. 


\section{References}

[1] See for example Statistical mechanics of membranes and surfaces, D. Nelson et al., eds (World Scientific, 1989).

[2] E. Browicz, Zbl. Med. Wiss. 28 (1890) 625.

[3] P. Nelson and T. Powers, "Renormalization of chiral couplings in tilted bilayer membranes," J. Phys. France II 3 (1993) 1535.

[4] P. Canham, J. Theor. Biol. 26 (1970) 61; W. Helfrich, Naturforsch. 28C (1973) 693.

[5] W. Cai and T.C. Lubensky, "Covariant hydrodynamics of fluid membranes", preprint (1993).

[6] P.G. de Gennes and C. Taupin, J. Phys. Chem. 86 (1982) 2294.

[7] W. Helfrich, J. Phys. (Paris) 46 (1985) 1263; ibid. 47 (1986) 321.

[8] L. Peliti and S. Leibler, "Effects of thermal fluctuations on systems with small surface tension," Phys. Rev. Lett. 54 (1985) 1690.

[9] D. Förster, "On the scale dependence, due to thermal fluctuations, of the elastic properties of membranes," Phys. Lett 114A (1986) 115.

[10] A. Polyakov, "Fine structure of strings," Nucl. Phys. B268 (1986) 406.

[11] H. Kleinert, "Thermal softening of curvature elasticity in membranes," Phys. Lett. 114A (1986) 263.

[12] H. Kleinert, "The membrane properties of condensing strings," Phys. Lett. 174B (1986) 335.

[13] J. Meunier, J. Phys. (France) 48 (1987) 1819.

[14] F. David, in [1].

[15] F. David and S. Leibler, "Vanishing tension of fluctuating membranes," J. Phys. II France 1 (1991) 959.

[16] D. Morse and S. Milner, "Fluctuations and phase behavior of surfactant vesicles," Preprint 1993.

[17] F. David, "Conformal field theories coupled to 2-D gravity in the conformal gauge," Mod. Phys. Lett. A3 (1988) 1651; J. Distler and H. Kawai, "Conformal field theory and 2-D quantum gravity," Nucl. Phys. B321 (1989) 509.

[18] J. Polchinski and A. Strominger, "Effective string theory," Phys. Rev. Lett. 67 (1991) 1681.

[19] M. Cates, "The Liouville field theory of random surfaces," Europhys. Lett. 8 (1988) 719.

[20] V. Popov, Functional Integrals in Quantum Field Theory and Statistical Mechanics (Riedel, 1983).

[21] A. Polyakov, Gauge fields and strings, (Harwood, 1987). 